\title{
Fermi-condensate quantum phase transition in high temperature superconductors
}

\author{
V.R. Shaginyan * \\ Petersburg Nuclear Physics Institute, Gatchina 188350, Russia
}

\begin{abstract}
The effect of a quantum phase transition associated with the appearance of the fermion condensate in an electron liquid on the properties of superconductors is considered. It is shown that the electron system in both superconducting and normal states exhibits characteristic features of a quantum protectorate after the point of this fermion-condensation quantum phase transition. The singleparticle spectrum of a superconductor can be represented by two straight lines corresponding to two effective masses $M_{F C}^{*}$ and $M_{L}^{*}$. The $M_{F C}^{*}$ mass characterizes the spectrum up to the binding energy $E_{0}$ and $M_{L}^{*}$ determines the spectrum at higher binding energies. Both effective masses are retained in the normal state. These results are used to explain the lineshape of single-particle excitations and other properties of high- $T_{c}$ superconductors and are in a good agreement with recent experimental data.
\end{abstract}

Keywords: Strongly correlated electrons; Superconductivity

Recent experiments gave rather accurate data on the dispersion of single-particle excitations over a wide range of binding energies [1]. These experiments were carried out with high- $T_{c}$ superconductors $\mathrm{Bi}_{2} \mathrm{Sr}_{2} \mathrm{CaCu}_{2} \mathrm{O}_{8+\delta}$ differing in the doping level both at temperatures $T$ below the critical temperature $T_{c}$ and at $T_{c} \leq T$. It was inferred that the dispersion of quasiparticle excitations can be described in the energy range $(-200-0) \mathrm{meV}$ by two straight lines intersecting at the binding energy $E_{0} \simeq(50-70)$ $\mathrm{meV}$ [1]. This circumstance directly points to the existence of a new energy scale in the self-energy part of quasiparticle excitations at temperatures $T \leq T_{c}$ and $T_{c} \leq T$ [1]. Therefore, new additional constraints can be imposed on the theories that are in principle applicable to the description of properties of high- $T_{c}$ superconductors. Experimental data on single-particle electron spectra of high- $T_{c}$ superconductors indicate that the perturbation of the superconducting phase and single-particle spectra by phonons, collective states, or impurities is very small. Therefore, this state can be described as a strongly collectivized quantum state or as "quantum protectorate" [2]. At $T=0$, the ground state energy $E_{g s}[\kappa(\mathbf{p}), n(\mathbf{p})]$ of two-dimensional electron liquid is a functional of the order parameter of the superconducting state $\kappa(\mathbf{p})$ and occupation numbers $n(\mathbf{p})$ and is determined by the known equation of the weak-coupling theory of superconductivity

$$
E_{g s}=E[n(\mathbf{p})]+\int V\left(\mathbf{p}_{1}, \mathbf{p}_{2}\right) \kappa\left(\mathbf{p}_{1}\right) \kappa^{*}\left(\mathbf{p}_{2}\right) \frac{d \mathbf{p}_{1} d \mathbf{p}_{2}}{(2 \pi)^{4}} .
$$

Here $E[n(\mathbf{p})]$ is the ground-state energy of the normal Fermi liquid, the pairing interaction $V\left(\mathbf{p}_{1}, \mathbf{p}_{2}\right)$ is assumed to be weak, while $n(\mathbf{p})=v^{2}(\mathbf{p})$, and $\kappa(\mathbf{p})=$ $v(\mathbf{p}) \sqrt{1-v^{2}(\mathbf{p})}$. Minimizing $E_{g s}$ with respect to $\kappa(\mathbf{p})$ we obtain the equation for the superconducting gap $\Delta(\mathbf{p})$

$$
\varepsilon-\mu=\Delta \frac{1-2 v^{2}(\mathbf{p})}{2 \kappa(\mathbf{p})} ; \Delta=-\int V\left(\mathbf{p}, \mathbf{p}_{1}\right) \kappa\left(\mathbf{p}_{1}\right) \frac{d \mathbf{p}_{1}}{4 \pi^{2}} .
$$

where the single-particle energy $\varepsilon(\mathbf{p})$ is determined by the Landau equation, $\varepsilon(\mathbf{p})=\delta E[n(\mathbf{p})] / \delta n(\mathbf{p}), \mu$ is chemical potential. If $V \rightarrow 0$, then, the gap $\Delta(\mathbf{p}) \equiv 0$, and Eq. (2) is reduced to the equation proposed in [3]

$$
\varepsilon(\mathbf{p})-\mu=0 \text {, if } 0<n(\mathbf{p})<1 ; p_{i} \leq p \leq p_{f} .
$$

This equation defines a Fermi liquid of a new type [1] for which the order parameter $\kappa(\mathbf{p})$ differs from zero in the $L_{F C}$ range of momenta $p_{i} \leq p \leq p_{f}$; the occupation numbers $n(\mathbf{p})=1,0$ outside the $L_{F C}$ range, as it must be in the normal Fermi liquid. It follows from Eq. (4), that a fermion system with the fermion condensate (FC) is broken into two quasiparticle subsystems: the first subsystem in the $L_{F C}$ range is occupied by the quasiparticles of the effective mass $M_{F C}^{*} \rightarrow \infty$, while the second one is occupied by quasiparticles of finite mass $M_{L}^{*}$ with momenta $p<p_{i}$. The FC appears in an electron system at a low density, when the effective electron-electron interaction constant is sufficiently large. In a common electron liquid, this constant is directly proportional to the dimensionless parameter $r_{s} \sim 1 / p_{F} a_{B}$, where $a_{B}$ is the Bohr radius and $p_{F}$ is the Fermi momentum. The FC occurs at a certain $r_{s}=r_{F C}<r_{c d w}$ and precedes the appearance of a charge-density wave (CDW) at $r_{s}=r_{c d w}$. This phase transition occurs at $T=0$ when the parameter $r_{s}$ attains its critical value $r_{F C}$ and represents a quantum phase transition. At $r_{s}>r_{F C}$ and $r_{s}-r_{F C} \ll r_{F C}$, the region $p_{f}-p_{i}$ occupied by the Fermi condensate is $\left(p_{f}-p_{i}\right) / p_{F} \sim r_{s}-r_{F C}$. Because the order parameter of the fermion-condensation quantum phase transition (FCQPT) is $\kappa(\mathbf{p})$, the maximum value of the gap $\Delta_{1} \sim V$, as it follows from Eq. (2), while $\kappa(\mathbf{p})$ is determined by the relatively strong particle-hole interaction. Therefore, the perturbation of the parameter $\kappa(\mathbf{p})$ by $V$ can be neglected in the first approximation. At $T=0, M_{F C}^{*}, E_{0}$, and $\Delta_{1}$ can be calculated from Eq. (2) [3]

*E-mail: vrshag@thd.pnpi.spb.ru 


$$
\begin{gathered}
\frac{p_{F}}{M_{F C}^{*}} \simeq \frac{2 \Delta_{1}}{p_{f}-p_{i}} ; \quad E_{0} \simeq 2 \frac{\left(p_{f}-p_{F}\right) p_{F}}{M_{F C}^{*}} \simeq 2 \Delta_{1} \\
\Delta_{1} \simeq 2 \frac{\beta \varepsilon_{F}\left(p_{f}-p_{F}\right)}{p_{F}} .
\end{gathered}
$$

Thus, electron system with the FC is characterized by two finite effective masses $M_{F C}^{*}$ and $M_{L}^{*}$, the single-particle dispersion at $p \sim p_{F}$ can be approximated by two straight lines, $E_{0}$ does not depend on $p_{f}-p_{i}$, and $M_{F C}^{*}$ is proportional to this difference. Taking the usual values of the dimensionless coupling constant $\beta \simeq V M_{L}^{*} /(2 \pi) \simeq 0.3$, $\left(p_{f}-p_{F}\right) / p_{F} \simeq 0.2$, we obtain from Eq. (4) that $\Delta_{1} \simeq 0.1 \varepsilon_{F}$, with $\varepsilon_{F}$ being the Fermi energy. Because $T_{c} \simeq \Delta_{1} / 2$, we conclude that $T_{c}$ reaches high values. At finite $T$, Eq. (4) is replaced by [3]

$$
\frac{p_{F}}{M_{F C}^{*}} \simeq \frac{4 T}{p_{f}-p_{i}} ; \quad E_{0} \simeq \frac{\left(p_{f}-p_{i}\right) p_{F}}{M_{F C}^{*}} \simeq 4 T \text {. }
$$

By comparing Eqs. (4) and (5), we see that $M_{F C}^{*}$ and $E_{0}$ weakly depend on temperature at $T \leq T_{c}$. If there exists the pseudogap above $T_{c}$, then $T_{c}$ is to be replaced by $T^{*}$ because in that case $2 \Delta_{1} \simeq T^{*}[3]$. The above consideration shows that the form of the single-particle spectrum $\varepsilon(\mathbf{p})$ is determined by the FCQPT and, therefore, its form is universal being almost independent of the contribution coming from impurities, phonons, etc. Actually, the particle-hole interaction defines only the region $L_{F C}$ occupied by the condensate after the point of the FCQPT. Thus, a system with the FC is characterized by a universal form of the single-particle spectrum and possesses quantum protectorate features at $T \ll T_{f}$, with $T_{f}$ being a temperature at which the effect of the FCQPT disappears [3]. Now turn to the description of the experimental data [1]. Experimental studies showed that the Van Hove singularity is located at the $(\pi, 0)$ point of the Brillouin zone, and an almost dispersionless section of the spectrum is observed in this region. This allows the suggestion to be made that the $\mathrm{FC}$ is arranged about this point. The line $Y \Gamma$, which is known as the line of zeros of the Brillouin zone, passes through the points $(\pi, \pi)$ $(0,0)$ at an angle of $\pi / 4$ to the line $Y \bar{M}$ passing through the points $(\pi, \pi)-(\pi, 0)$. Single-particle spectra were measured along the lines parallel to $Y \Gamma$ and $Y \bar{M}$, from the line of zeros to the $Y \bar{M}$ line. It was shown that $E_{0}$ is constant for a given sample being independent of the angle $\phi$, reckoned from $Y \Gamma$ to $Y \bar{M}$. The angle (kink) between the straight line characterizing the single-particle spectrum grows with increasing $\phi$ and with decreasing doping level. This general pattern is retained at $T>T_{c}$ [1]. To describe these experimental data, we use the following results: $r_{s}$ grows with decreasing the doping level and exceeds the critical value $r_{F C}$ in the overdoping region; the difference $\left(p_{f}\left(r_{s}\right)-p_{i}\left(r_{s}\right)\right)$ as a function of $r_{s}$ grows with increasing $r_{s}$; the difference $\left(p_{f}(\phi)-p_{i}(\phi)\right)$ increases with increasing $\phi$ and attains a maximum at $(\pi, 0)$. Noticing that strong fluctuations of the charge density or CDW are observed in undoped samples, we may conclude that the formation of the FC in copper oxides is a quite determinate process stemming from the general properties of a low-density electron liquid. Then, Eq. (4) shows that $E_{0}$ does not depend on $\phi$ and $T$ at $T \leq T_{c}$, while the kink increases with increasing $\phi$, because $M_{F C}^{*}$ linearly depends on $\left(p_{f}(\phi)-p_{i}(\phi)\right)$. Then, $E_{0} \simeq(50-70) \mathrm{meV}[1]$, which is in agreement with Eq. (4), because $E_{0} \simeq 2 \Delta_{1}$ in these materials. Then, the dispersion kink must increase with decreasing doping level. As it follows from Eq. (4), $E_{0} \simeq 2 \Delta_{1} \sim\left(p_{f}-p_{F}\right)$, the kink point shifts towards higher binding energies as the doping level decreases. Consider the lineshape $L(q, \omega)$ being a function of two variables. Measurements carried out at a fixed binding energy $\omega=\omega_{0}$, where $\omega_{0}$ is the energy of a single-particle excitation, determine the lineshape $L\left(q, \omega=\omega_{0}\right)$ as a function of momentum $q$. Eqs. (4) and (5) show that $M_{F C}^{*}$ is finite and the system behaves as a normal liquid at the energies $\omega<2 \Delta_{1}$, while the width of single-particle excitations is of the order of $T$ [3]. This behavior was observed in experiments on measuring the lineshape at a fixed energy [1]. The lineshape can also be determined as a function $L\left(q=q_{0}, \omega\right)$ at a fixed $q=q_{0}$. At small $\omega, L\left(q=q_{0}, \omega\right)$ has a characteristic maximum and width. At energies $\omega \geq 2 \Delta_{1}$, quasiparticles of mass $M_{L}^{*}$ come into play, leading to a growth of the function. One may also use the Kramers-Krönig transformation to construct the imaginary part of the self-energy starting with the real one. As a result, the lineshape $L\left(q=q_{0}, \omega\right)$ possesses the known peak-dip-hump structure directly defined by the existence of the two effective masses $M_{F C}^{*}$ and $M_{L}^{*}$. All these results are in good qualitative agreement with the experimental data [1]. This work was supported by the Russian Foundation for Basic Research, project no. 01-02-17189.

[1] T. Valla et al., Science 285, 2110 (1999); P.V. Bogdanov et al., Phys. Rev. Lett. 85, 2581 (2000); A. Kaminski et al., Phys. Rev. Lett. 86, 1070 (2001).

[2] R.B. Laughlin and D. Pines, Proc. Natl. Acad. Sci. USA 97(1), 28 (2000); P.W. Anderson, cond-mat/0007185, cond-mat/0007287.

[3] V.A. Khodel and V.R. Shaginyan, JETP Lett. 51, 553 (1990); S.A. Artamonov and V.R. Shaginyan, JETP 92, 287 (2001); M.Ya. Amusia and V.R. Shaginyan, Phys. Rev. B 63, 224507 (2001).

[4] G. E. Volovik, JETP Lett. 53, 222 (1991). 